\title{
Phase 2 non-randomised trial of secondary cytoreduction and hyperthermic intraperitoneal chemotherapy in recurrent platinum-sensitive ovarian cancer
}

\author{
Hemanth Raj ${ }^{1 \dagger}$, Marri Sri Santosh Keerthi ${ }^{1 \dagger}$, Ravisankar Palaniappan ${ }^{2}$, Ujwala Prakash ${ }^{1}$, Manikandan Dhanushkodi ${ }^{3, a}$ iD and \\ Trivadi S Ganesan ${ }^{3}$ \\ ${ }^{1}$ Department of Surgical Oncology, Cancer Institute (WIA), 38, Sardar Patel Road, Chennai 600036, Tamil Nadu, India \\ ${ }^{2}$ Department of Surgical Oncology, Sri Venkateshwaraa Medical College Hospital and Research Centre, Puducherry 605102, India \\ ${ }^{3}$ Department of Medical Oncology, Cancer Institute (WIA), 38, Sardar Patel Road, Chennai 600036, Tamil Nadu, India \\ tHemanth Raj and Marri Sri Santosh Keerthi contributed equally. \\ ahttps://orcid.org/0000-0002-8192-3856
}

\section{Abstract}

Background: The role of secondary cytoreduction with hyperthermic intraperitoneal chemotherapy (HIPEC) is not clearly defined in recurrent platinum-sensitive ovarian cancer (PSOC). There is a paucity of studies on secondary cytoreduction with HIPEC in PSOC from developing countries like India. This study was done to assess the feasibility and safety of secondary cytoreduction and HIPEC in recurrent PSOC.

Methods: This was a prospective, non-randomised, open-label, phase 2 trial of secondary cytoreduction and HIPEC (Cisplatin $75 \mathrm{mg} / \mathrm{m}^{2} 43^{\circ} \mathrm{C}$ over 60 minutes) in patients with recurrent platinum-sensitive epithelial carcinoma of ovary/fallopian tube/peritoneum done in a tertiary cancer centre from February 2016 to August 2019. The primary outcome was to assess the overall survival (OS) and the secondary outcomes were to assess the progression-free survival (PFS) and toxicity.

Results: Twenty-seven patients were screened and among them, 15 patients were included in this analysis with a median follow-up of 25 months. The mean cancer antigen (CA) 125 at the time of recurrence was $149 \mathrm{U} / \mathrm{mL}$ (range: 10-2,030 U/mL) and the median platinum-free interval was 21 months. The perioperative chemotherapy used was paclitaxel + carboplatin 53.3\% (8/15), liposomal doxorubicin + carboplatin 40\% (6/15) and none $6.5 \%$ (1/15). The median Peritoneal Carcinomatosis Index score was 8 (range: $3-25)$. The Clavien Dindo score was I, II and III in 6.7\%, $26.7 \%$ and $13.3 \%$ patients, respectively. Recurrence was radiological and biochemical in 60\% (9/15) and 7\% (1/15), respectively. The most common site of recurrence was intra-abdominal (peritoneal). The median PFS and OS were 15 months (range: 0-34) and 26 months (range: 23-29), respectively. The grade 3 or 4 toxicity was $40 \%$.

Conclusion : Secondary cytoreduction with HIPEC is feasible and safe in recurrent PSOC. Conclusive evidence that secondary cytoreduction with HIPEC is essential awaits the results from ongoing randomised controlled trials.

Keywords: HIPEC, platinum-sensitive, recurrent ovarian cancer, secondary cytoreduction
Correspondence to: Manikandan Dhanushkodi Email: dmani1982@gmail.com

ecancer 2021, 15:1260

https://doi.org/10.3332/ecancer.2021.1260

Published: 05/07/2021

Received: 11/04/2021

Publication costs for this article were supported by ecancer (UK Charity number 1176307).

Copyright: (c) the authors; licensee ecancermedicalscience. This is an Open Access article distributed under the terms of the Creative Commons Attribution License (http:// creativecommons.org/licenses/by/4.0), which permits unrestricted use, distribution, and reproduction in any medium, provided the original work is properly cited. 


\section{Background}

Epithelial ovarian carcinoma (EOC) being an indolent disease is often diagnosed at an advanced stage. The standard treatment for advanced ovarian carcinoma is primary cytoreduction followed by adjuvant platinum-based doublet chemotherapy. Despite being chemo-sensitive, $70 \%$ of patients have a recurrence during the first 3 years of follow-up with a dismal 5 -year overall survival (OS) of 20\%-30\% [1]. The peritoneum is the most common site of recurrence in advanced ovarian cancer [2].

Cytoreduction with hyperthermic intraperitoneal chemotherapy (HIPEC) has been shown to improve survival in first line treatment of advanced ovarian cancer [3]. In patients with recurrent disease, secondary cytoreduction has been shown to improve survival based on many retrospective studies [4]. Factors (Arbeitsgemeinschaft Gynaekologische Onkologie (AGO) criteria) associated with improved survival after secondary cytoreduction are Eastern Cooperative Oncology Group (ECOG) performance status 0, absence of ascites > $500 \mathrm{~mL}$, Federation of Gynecology and Obstetrics (FIGO) stage I and II at diagnosis and no residual tumour after primary surgery [5]. A phase 3 trial of secondary cytoreduction in platinum-sensitive ovarian cancer (PSOC) based on the AGO score has been shown to improve progression-free survival (PFS) by 5 months [6]. However, another phase 3 randomised controlled study [Gynecologic Oncology Group (GOG) 213] has shown that secondary cytoreduction does not improve PFS and OS in platinum-sensitive recurrent ovarian cancer [7]. Thus, the role of secondary cytoreduction in recurrent EOC remains uncertain.

There are several reports in the literature evaluating the role of HIPEC following surgery for recurrent ovarian cancer. There are at least two reports from Italy [8, 9] showing improvement in survival in recurrent disease. In one report, the median PFS was 27 months, while OS was 70 months. Similarly, a randomised trial from Greece [10] showed the HIPEC with maximal cytoreductive surgery (CRS) improved survival over surgery alone. Currently, there are at least two ongoing randomised trials (HORSE and CHIPOR) evaluating the role of HIPEC in recurrent ovarian cancer [11].

In India, there have been reports evaluating the role of HIPEC in peritoneal carcinomatosis that is amenable to surgery [12-14]]. However, there have been no randomised trials as randomised phase 3 trials will need many patients that need multicentre collaboration.

This study was done to assess the feasibility and safety of secondary cytoreduction and HIPEC in recurrent PSOC.

\section{Methods}

\section{Participants \& settings}

This was a prospective, non-randomised, open-label, phase 2 trial of secondary cytoreduction and HIPEC in patients with platinum-sensitive recurrent epithelial carcinoma of ovary/fallopian tube/peritoneum done in a tertiary cancer centre from February 2016 to August 2019.

The inclusion criteria were consecutive recurrent PSOC (platinum-free interval $>6$ months), age $>18$ years, ECOG performance status < 2 , absolute neutrophil count $>1,500 / \mathrm{mm}^{3}$, platelets $>1,50,000 / \mathrm{mm}^{3}$, international normalised ratio $<1.5$, serum total bilirubin $<1.5 \mathrm{mg} / \mathrm{dL}$, serum alkaline phosphatase $<2.5$ times the upper limit of normal, normal renal functions and disease amenable for secondary cytoreduction. The exclusion criteria were patients with bowel obstruction, absence of peritoneal disease and non-epithelial/borderline ovarian cancer.

\section{Interventions}

The preoperative evaluation included serum cancer antigen (CA) 125, chest X-ray and contrast-enhanced computed tomography (CECT) of abdomen/pelvis. All patients underwent planned perioperative chemotherapy for a total of six cycles using carboplatin-based doublet (paclitaxel/liposomal doxorubicin).

The surgery performed was secondary cytoreduction including resection of the recurrent tumour along with the peritoneum (including central peritoneal compartment, greater omentectomy, splenectomy, peritonectomy beneath both hemi-diaphragms, removal of tumour from 
the liver surface and pelvic peritonectomy). Bowel resection and anastomosis were done as required and intra-abdominal drains were placed. Temporary colostomy was done in cases where rectum was resected. Peritoneal Carcinomatosis Index (PCI) and completeness of cytoreduction scores were recorded. The skin incision was closed after placing inflow and outflow catheters for administering HIPEC.

HIPEC was delivered using EXIPER M03223, Menfis division, MEDICA S.P.A, Italy till February 2018 and thereafter using Performer HT, RanD, Medolla, Italy. The perfusion circuit consisted of two inflow catheters placed in the upper abdomen, two outflow catheters placed in the pelvis, a roller pump and a heat exchanger. Temperature probes were attached to inflow and outflow catheters. The premedication before HIPEC with cisplatin was injection (Inj) fosaprepitant $150 \mathrm{mg}$ and Inj. palonosetron $0.25 \mathrm{mg}$ intravenously, 30 minutes before cisplatin. Cisplatin was infused at a rate of $75 \mathrm{mg} / \mathrm{m}^{2}$ in $2 \mathrm{~L} / \mathrm{m}^{2}$ peritoneal dialysis fluid at a temperature of $43^{\circ} \mathrm{C}$ over 60 minutes after attaining the minimum temperature of $42^{\circ} \mathrm{C}$ throughout the abdominal cavity, with a flow rate of $1,000 \mathrm{~mL} /$ minute.

During the post-operative period, patients were not fed orally till Ryle's tube aspiration was $<100 \mathrm{~mL}$. Patients received antibiotics (Inj. cefuroxime 1,500 mg IV and Inj. metronidazole $500 \mathrm{mg}$ IV for 3 days), proton pump inhibitors, prophylaxis for deep vein thrombosis (low molecular weight heparin, pneumatic calf pump) and analgesics. Abdominal drains were removed when the output was $<50 \mathrm{~mL}$. Patients were discharged when they were on a normal diet, drains removed, wound healed and sutures removed. The patients were followed up every 3 monthly for the 2 years, 6 monthly in the 3rd and 4th year and annually thereafter. During follow-up, patients underwent history, clinical examination and serum CA-125. CECT of abdomen/pelvis was performed in patients with rising CA-125 or when clinically indicated. The patient demographics, pre-operative investigations, intra-operative findings, complications (intraoperative and postoperative) and follow-up data were recorded.

\section{Statistical analysis}

The PFS was calculated from the date of HIPEC until the date of progression or date of death or date of last follow-up. The OS was calculated from the date of HIPEC until the date of death or date of last follow-up. The survival was estimated using the Kaplan-Meier method. Data were analysed using SPSS, version 23 (SPSS Inc., IL, USA) and $p$-value of $<0.05$ was considered significant.

Written informed consent was taken from all the patients. The study was conducted according to various guidelines for the ethical conduct of studies including the good clinical practice guidelines, and the Indian Council of Medical Research. This study was in accordance with the ethical standards of the responsible committee on human experimentation (institutional or regional) and with the Helsinki Declaration of 1964, as revised in 2013. This trial was approved by Drug Controller General of India (DCGI), Scientific Advisory Committee (SAC), Institutional Ethics Committee (IEC) and registered in Clinical Trials Registry of India (CTRI).

\section{Results}

Twenty-seven patients (Figure 1) underwent screening among whom twelve were excluded from the trial. The reasons for exclusion were incomplete first surgery $(n=1)$, inoperable disease $(n=7)$, presence of ascites $(n=2)$, platinum-resistant disease $(n=1)$ and inability to achieve optimal cytoreduction $(n=1)$. Fifteen patients were included in this trial with a median follow-up of 25 months (Table 1). The median age was 51 years (range: $42-70$ years). Co-morbid illness was present in 53\% (8/15), the most common being diabetes (40\%) followed by hypertension (13\%). A family history of cancer was present in $26 \%(4 / 15)$, and the most common malignancy was breast cancer (13\%). About $53 \%$ were postmenopausal and the rest were premenopausal, all women were ECOG performance status 1.

The stage at the initial diagnosis of ovarian carcinoma was III (80\%) or IV (20\%). The histology was serous adenocarcinoma, poorly differentiated adenocarcinoma and clear cell carcinoma in $73 \%, 20 \%$ and $7 \%$, respectively. The differentiation was low to intermediate in $7 \%$ and high grade in $93 \%$. The median CA 125 at presentation was $2,833 \mathrm{U} / \mathrm{mL}$ (range: $15-9,730 \mathrm{U} / \mathrm{mL}$ ). All patients received neoadjuvant chemotherapy. The majority $(93 \%, n=14)$ received paclitaxel and carboplatin and one patient $(7 \%)$ received liposomal doxorubicin and carboplatin. Pathological complete response was achieved in one patient (7\%). All patients underwent optimal interval cytoreduction. After completion of six cycles of paclitaxel carboplatin, $73 \%$ of patients received one cycle of intraperitoneal cisplatin $(100 \mathrm{mg})$. The median duration of remission after completion of all treatment was 22 months (range: 8-76 months). 


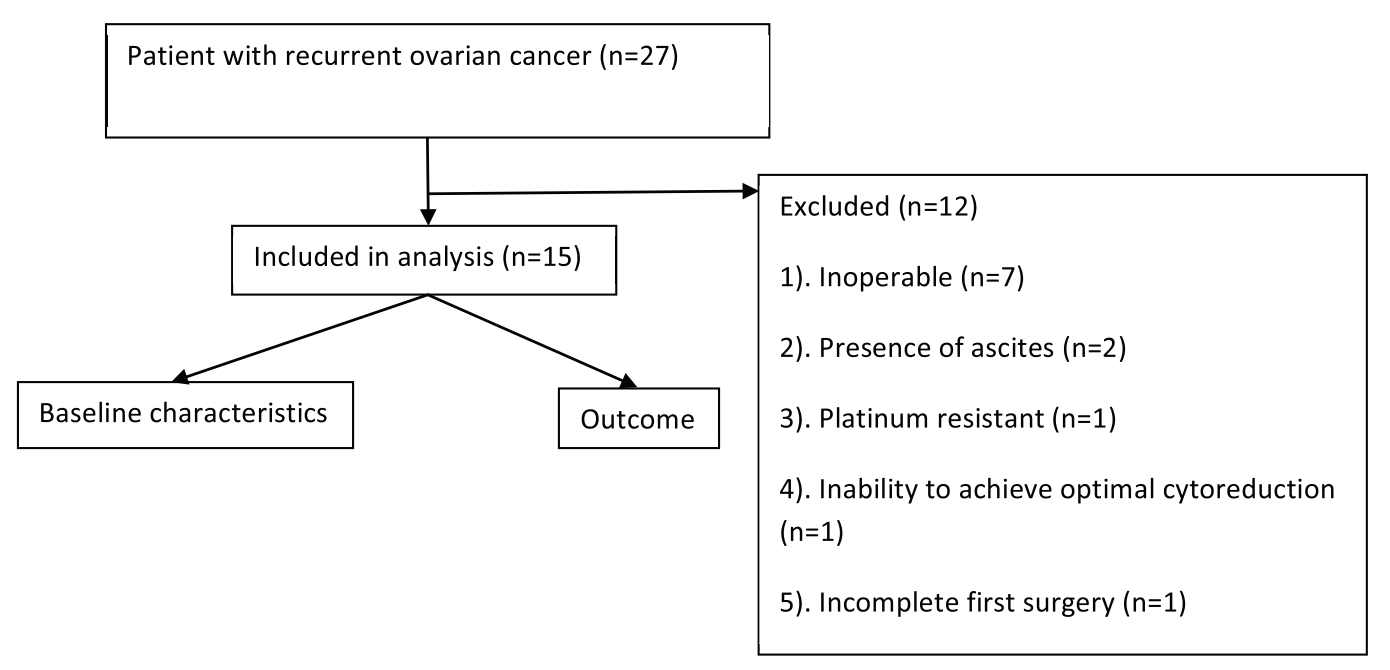

Figure 1. Study flowchart.

The mean CA 125 at the time of recurrence was $149 \mathrm{U} / \mathrm{mL}$ (range: 10-2,030 U/mL) and the median platinum-free interval was 21 months. The perioperative chemotherapy used was paclitaxel + carboplatin 53.3\% (8/15), liposomal doxorubicin + carboplatin 40\% (6/15) and none $6.5 \%(1 / 15)$. The median $\mathrm{PCl}$ score before secondary cytoreduction was 8 (range: $3-25)$. The mean duration of surgery (secondary cytoreduction and HIPEC) was $455+85$ minutes (Table 2). Following surgery, blood component support was required in 60\% (9/15), total parenteral nutrition (TPN) in 87\% (13/15), inotropes in 80\% (12/15) and mechanical ventilation in 47\% (7/12) patients. The median time to ambulation was the 4th postoperative day. The median duration of hospital stay was 13 days (range: $8-18$ ). The nadir white blood cell count was 3,500 cells $/ \mathrm{mm}^{3}$ and none of them had nephrotoxicity. The Clavien Dindo score was I, II and III in $6.7 \%, 26.7 \%$ and $13.3 \%$ patients, respectively.

Ten patients have had a recurrence on follow-up. Recurrence was radiological and biochemical in 60\% (9/15) and 7\% (1/15), respectively. The most common site of recurrence after secondary cytoreduction and HIPEC was intra-abdominal (peritoneal). Thirteen patients were evaluable for survival analysis. The median PFS (Figure 2) and OS (Figure 3) were 15 months (range: 1-34) and 26 months (range: 23-29), respectively. The most common systemic therapy for recurrent disease after secondary cytoreduction and HIPEC was liposomal doxorubicin carboplatin followed by oral etoposide. At this time of follow-up, two patients have died due to progressive disease.

\section{Discussion}

The rationale for using HIPEC in ovarian cancer is that peritoneum is the most common site of recurrence, and HIPEC increases the drug concentration in the peritoneal cavity [15]. Moreover, hyperthermia causes direct cytotoxicity to cancer cells and is synergistic with chemotherapy [16]. Also, secondary CRS acts synergistically by reducing the bulk of the disease and thereby improving drug penetration and reducing the risk of further chemo-resistance.

The morbidity of the combined approach ranges from $20 \%$ to $66 \%$ and has been largely attributed to the extensive surgery required in an already previously operated patient. In the present study, the incidence of grade II complications and more was $40 \%$ as seen in other studies. The median duration of stay after cytoreduction and HIPEC of 8-25 days was previously reported which is also similar to our trial (median: 13 days, range: $8-18$ days) [17]. 
Table 1. Baseline characteristics.

\begin{tabular}{|c|c|c|}
\hline \multicolumn{3}{|c|}{ Demographics $(n=15)$} \\
\hline Age in years & Median (range) & $51(42-70)$ \\
\hline Co-morbid illness & Absent/present & $8(53 \%) / 7(47 \%)$ \\
\hline Family history of malignancy & Absent/present & $11(73.3 \%) / 4(26.7 \%)$ \\
\hline Menopausal status & Pre-menopausal/post-menopausal & $7(47 \%) / 8(53 \%)$ \\
\hline \multicolumn{3}{|c|}{ Variables at initial diagnosis $(n=15)$} \\
\hline CA-125 at diagnosis $(\mathrm{U} / \mathrm{mL})$ & Median (range) & $2833(15.1-9,730)$ \\
\hline Stage at diagnosis & III/IV & $12(80 \%) / 3(20 \%)$ \\
\hline Tumour histology & $\begin{array}{l}\text { Serous cystadenocarcinoma/clear } \\
\text { cell carcinoma/poorly differentiated } \\
\text { adenocarcinoma }\end{array}$ & $11(73.3 \%) / 1(6.7 \%) / 3(20 \%)$ \\
\hline Tumour grade & Low or intermediate/high grade & $1(6.7 \%) / 14(93.3 \%)$ \\
\hline Intravenous chemotherapy & Paclitaxel + carboplatin/others & $14(93.3 \%) / 1(6.7 \%)$ \\
\hline Intra-peritoneal chemotherapy & Received & $11(73 \%)$ \\
\hline \multicolumn{3}{|c|}{ Variables at relapse / recurrence $(n=15)$} \\
\hline CA-125 at relapse $(\mathrm{U} / \mathrm{mL})$ & Median (range) & $149(10.5-2,030)$ \\
\hline Chemotherapy type & $\begin{array}{l}\text { Paclitaxel + carboplatin/liposomal doxorubicin } \\
+ \text { carboplatin/none }\end{array}$ & $8(53.3 \%) / 6(40 \%) / 1$ (6.7\%) \\
\hline $\mathrm{PCl}$ score & Median (range) & $8(3-25)$ \\
\hline
\end{tabular}

Table 2. Treatment, toxicity \& survival $(n=15)$.

\begin{tabular}{|c|c|c|}
\hline Duration of surgery (minutes) & Mean $+2 \mathrm{SD}$ & $455+85$ \\
\hline Grade of toxicity ${ }^{a}$ & $\mathrm{I} / \mathrm{II}$ & $11(73.3 \%) / 4(26.7 \%)$ \\
\hline Blood transfusion & & $9(60 \%)$ \\
\hline Intra-operative hypotension & & $12(80 \%)$ \\
\hline Post-operative ventilator support & & $7(46.7 \%)$ \\
\hline Post-operative TPN & & $13(86.7 \%)$ \\
\hline Duration of post-operative stay (days) & Median (range) & $13(8-18)$ \\
\hline Clavien Dindo score & O/I/II/III & $\begin{array}{c}8(53.3 \%) / 1(6.7 \%) / 4(26.7 \%) / 2 \\
(13.3 \%)\end{array}$ \\
\hline Peri-operative chemotherapy & Received/not received & $9(60 \%) / 6(40 \%)$ \\
\hline \multicolumn{3}{|l|}{ Follow-up $(n=13)$} \\
\hline Relapse & & $10(76.9 \%)$ \\
\hline Mortality & Alive/expired & $11(85 \%) / 2(15 \%)$ \\
\hline PFS in months ${ }^{b}$ & Median (range) & $15(1-34.5)$ \\
\hline OS in months ${ }^{b}$ & Median (range) & $26(22.9-29.0)$ \\
\hline
\end{tabular}

according to the Common Terminology Criteria for Adverse Events

${ }^{b}$ Calculated using Kaplan-Meir analysis

SD, Standard deviation; TPN, Total parenteral nutrition 


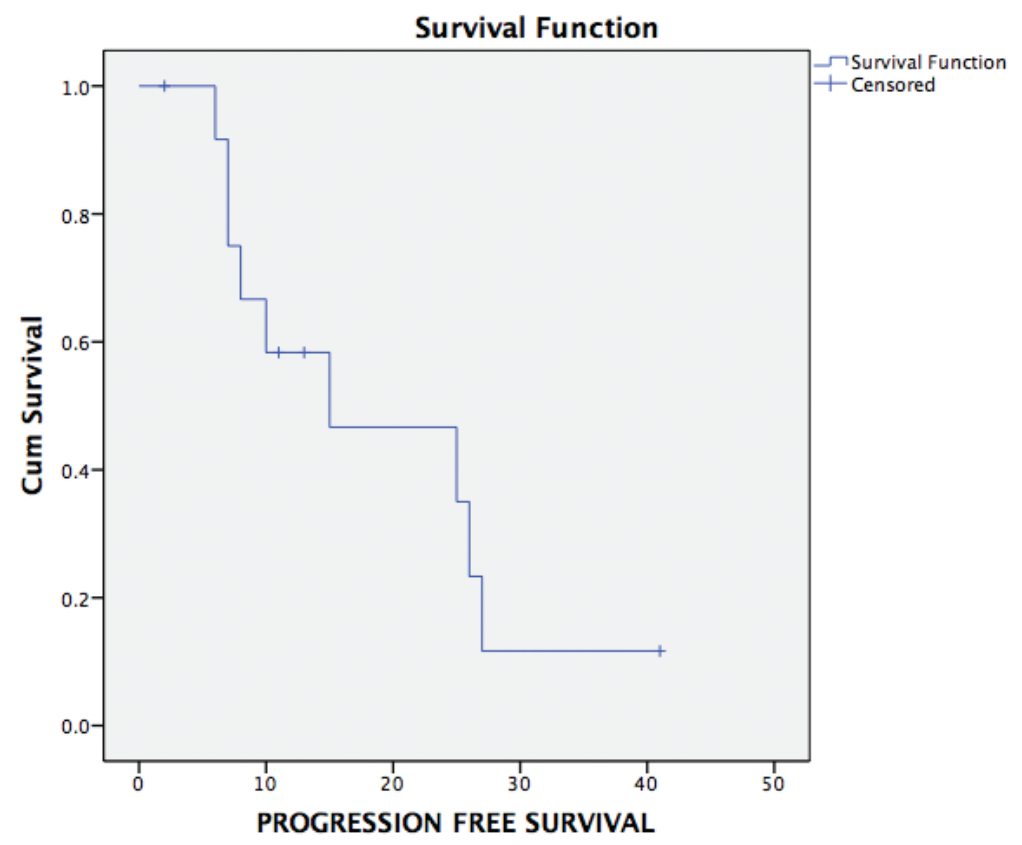

Figure 2. Kaplan-Meier analysis showing PFS of 13 patients with platinum sensitive recurrent ovarian cancer who underwent secondary cytoreduction with HIPEC. The median PFS was 15 months (range: 1-34 months).

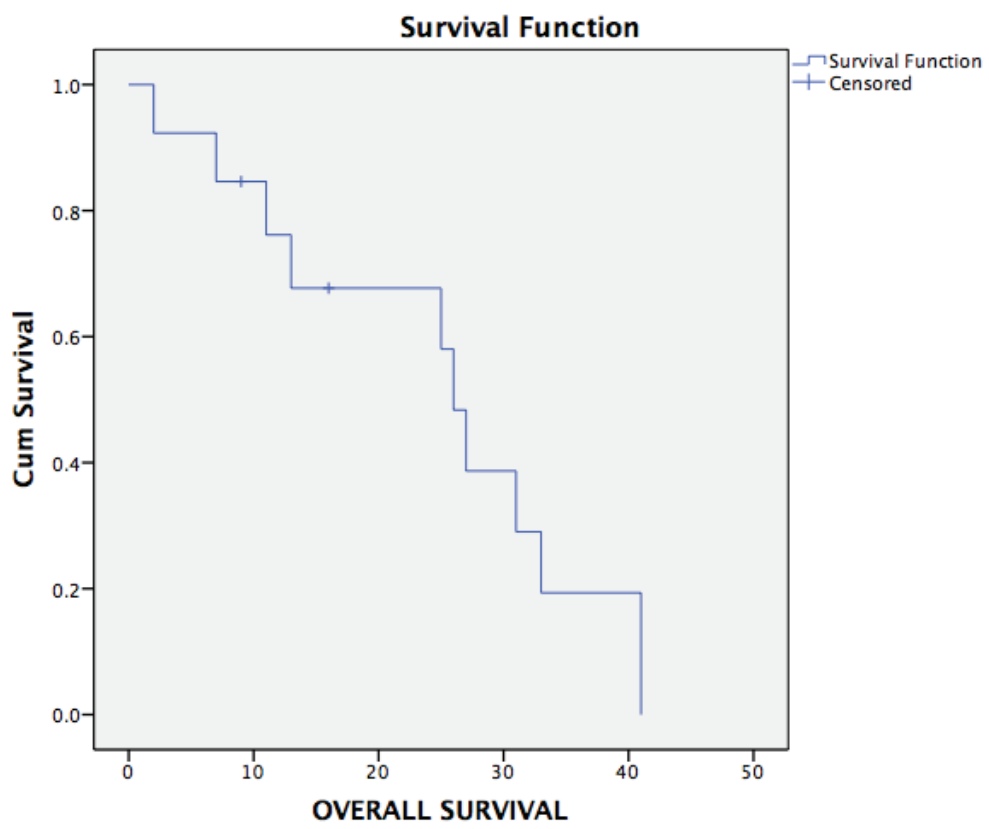

Figure 3. Kaplan-Meier analysis showing OS of 13 patients with platinum sensitive recurrent ovarian cancer who underwent secondary cytoreduction with HIPEC. The median OS was 26 months (range: 22.9-29.0). 
The burden of peritoneal carcinomatosis determined by the $\mathrm{PCl}$ score [18] and the extent of cytoreduction achieved significantly influence the survival rates. Some studies have used $\mathrm{PCl}$ scores in identifying patients with limited peritoneal cancer indicating that those with a lesser $\mathrm{PCl}$ score could benefit more with the combined approach. A Chinese study of 40 patients showed that patients with $\mathrm{PCl} \leq 20$ had better OS than those with $\mathrm{PCl}>20$ (76.6 versus 38.5 months, $p=0.01$ ) [19]. In this trial, the median PCl was 8 (range: $3-25$ ) which shows that our subset of patients could benefit more with CRS and HIPEC. Mulier et al [20] reported that complete cytoreduction was associated with a median OS and 5-year OS of 97.4 months and $63 \%-67 \%$, respectively.

A phase 3 trial of 120 patients from Greece showed that cytoreduction with HIPEC improved survival as compared to only cytoreduction in patients with recurrent ovarian cancer. However, this study was criticised for inappropriate patient selection and statistical analysis [10]. A multicentre French study of 566 patients with advanced ovarian cancer showed improved survival with cytoreduction and HIPEC [21]. An Italian study with 12 patients showed that tertiary cytoreduction with HIPEC repetition is feasible [22].

The systemic therapy that has improved PFS in PSOC includes targeted therapy (bevacizumab) [23] based chemotherapy, Poly ADP Ribose Polymerase (PARP) inhibitors like olaparib [24], niraparib [25] and rucaparib [26]. Immunotherapy with pembrolizumab is an option in patients with microsatellite instability-high or mismatch repair deficient tumours [27]. In this trial, the median PFS and OS were 15 months and 26 months, respectively.

The strengths of this study are the prospective design and the first HIPEC study in Indian patients with recurrent ovarian cancer. The limitations include small sample size, single institutional study, non-randomised design, lack of breast cancer status and non-usage of targeted therapy (bevacizumab) and PARP inhibitors. The results of the phase 3 randomised controlled trials will throw more light on the efficacy of secondary cytoreduction and HIPEC.

\section{Conclusion}

Secondary cytoreduction with HIPEC is feasible and safe in recurrent PSOC. Conclusive evidence that secondary cytoreduction with HIPEC is essential awaits the results from ongoing randomised controlled trials.

\section{Funding}

This trial was supported by Cancer Institute (WIA), Chennai.

\section{Conflicts of interest}

The authors declare that they have no conflicts of interest.

\section{Authors' contributions}

Conception: Hemanth Raj, Ravisankar Palaniappan, Trivadi S Ganesan. Acquisition: Marri Sri Santosh Keerthi, Hemanth Raj, Ujwala Prakash, Manikandan Dhanushkodi. Analysis: Marri Sri Santosh Keerthi, Manikandan Dhanushkodi, Trivadi S Ganesan. All authors made substantial contributions towards drafting and final approval, and they agree to be accountable on all aspects of the manuscript.

\section{Acknowledgments}

None. 


\section{Affiliations}

None.

\section{Details of earlier presentation}

None.

\section{Institutional review board approval}

All procedures performed in studies involving human participants were in accordance with the ethical standards of the IEC and with the 1964 Helsinki Declaration and its later amendments or comparable ethical standards. The study was approved by the SAC on 03/01/2015, IEC on 21/02/2015, DCGI on 29/01/16 and CTRI on 04/09/2017.

\section{References}

1. Hennessy BT, Coleman RL and Markman M (2009) Ovarian cancer Lancet 374 1371-1382 https://doi.org/10.1016/S01406736(09)61338-6 PMID: 19793610

2. Amate P, Huchon C, and Dessapt AL, et al (2013) Ovarian cancer: sites of recurrence Int J Gynecol Cancer 23 1590-1596 https://doi. org/10.1097/IGC.0000000000000007 PMID: 24172095

3. van Driel WJ, Koole SN, and Sikorska K, et al (2018) Hyperthermic intraperitoneal chemotherapy in ovarian cancer N Engl J Med 378 230-240 https://doi.org/10.1056/NEJMoa1708618 PMID: 29342393

4. Gockley A, Melamed A, and Cronin A, et al (2019) Outcomes of secondary cytoreductive surgery for patients with platinum-sensitive recurrent ovarian cancer Am J Obstet Gynecol 221 625.e1-625.e14 https://doi.org/10.1016/j.ajog.2019.06.009

5. Harter P, du Bois A, and Hahmann M, et al (2006) Surgery in recurrent ovarian cancer: the Arbeitsgemeinschaft Gynaekologische Onkologie (AGO) DESKTOP OVAR trial Ann Surg Oncol 13 1702-1710 https://doi.org/10.1245/s10434-006-9058-0 PMID: 17009163

6. Du Bois A, Vergote I, and Ferron G, et al (2017) Randomized controlled phase III study evaluating the impact of secondary cytoreductive surgery in recurrent ovarian cancer: AGO DESKTOP III/ENGOT ov20 J Clin Oncol 35 5501-5501 https://doi.org/10.1200/ JCO.2017.35.15_suppl.5501

7. Coleman RL, Spirtos NM, and Enserro D, et al (2019) Secondary surgical cytoreduction for recurrent ovarian cancer N Engl J Med 381 1929-1939 https://doi.org/10.1056/NEJMoa1902626 PMID: 31722153 PMCID: 6941470

8. Fagotti A, Costantini B, and Petrillo M, et al (2012) Cytoreductive surgery plus HIPEC in platinum-sensitive recurrent ovarian cancer patients: a case-control study on survival in patients with two year follow-up Gynecol Oncol 127 502-505 https://doi.org/10.1016/j. ygyno.2012.09.020 PMID: 23022234

9. Petrillo M, De laco P, and Cianci S, et al (2016) Long-term survival for platinum-sensitive recurrent ovarian cancer patients treated with secondary cytoreductive surgery plus hyperthermic intraperitoneal chemotherapy (HIPEC) Ann Surg Oncol 23 1660-1665 https://doi. org/10.1245/s10434-015-5050-x

10. Spiliotis J, Halkia E, and Lianos E, et al (2015) Cytoreductive surgery and HIPEC in recurrent epithelial ovarian cancer: a prospective randomized phase III study Ann Surg Oncol 22 1570-1575 https://doi.org/10.1245/s10434-014-4157-9 
11. Cowan RA, O'Cearbhaill RE, and Zivanovic O, et al (2017) Current status and future prospects of hyperthermic intraoperative intraperitoneal chemotherapy (HIPEC) clinical trials in ovarian cancer Int J Hyperthermia 33 548-553 https://doi.org/10.1080/02656736.2017 .1283066 PMID: 28092994 PMCID: $\underline{5776684}$

12. Padmanabhan N, Kumar BR, and Pookunju AP, et al (2015) Preliminary experience and morbidity analysis of cytoreductive surgery with hyperthermic intraperitoneal chemotherapy (CRS/HIPEC) from a Tertiary Cancer Center in India J Clin Diagn Res 9 XC09 PMID: 26266201 PMCID: 4525591

13. Somashekhar SP, Ashwin KR, and Yethadka R, et al (2019) Impact of extent of parietal peritonectomy on oncological outcome after cytoreductive surgery and HIPEC Pleura Peritoneum 420190015 https://doi.org/10.1515/pp-2019-0015 PMID: 31799371 PMCID: 6881664

14. Jagannath $P$, Dharmadhikari N, and Shah $R$ (2014) Initial experience with hyperthermic intra peritoneal chemotherapy and cytoreductivesurgery Indian J Cancer 51189 https://doi.org/10.4103/0019-509X.138304 PMID: 25104207

15. Spratt JS, Adcock RA, and Sherrill W, et al (1980) Clinical delivery system for intraperitoneal hyperthermic chemotherapy Cancer Res 40 256-260 PMID: 6766084

16. González-Moreno S (2010) Hyperthermic intraperitoneal chemotherapy: rationale and technique World J Gastrointest Oncol 2 68 https://doi.org/10.4251/wjgo.v2.i2.68 PMID: 21160924 PMCID: 2999165

17. Chua TC, Robertson G, and Liauw W, et al (2009) Intraoperative hyperthermic intraperitoneal chemotherapy after cytoreductive surgery in ovarian cancer peritoneal carcinomatosis: systematic review of current results J Cancer Res Clin Oncol 135 1637-1645 https:// doi.org/10.1007/s00432-009-0667-4 PMID: 19701772

18. Jónsdóttir B, Lomnytska M, and Poromaa IS, et al (2021) The peritoneal cancer index is a strong predictor of incomplete cytoreductive surgery in ovarian cancer Ann Surg Oncol 28 244-251 https://doi.org/10.1245/s10434-020-08649-6

19. Sun JH, Ji ZH, and Yu Y, et al (2016) Cytoreductive surgery plus hyperthermic intraperitoneal chemotherapy to treat advanced/recurrent epithelial ovarian cancer: results from a retrospective study on prospectively established database Transl Oncol $9130-138$ https:// doi.org/10.1016/j.tranon.2016.02.002 PMID: 27084429 PMCID: 4833965

20. Mulier S, Claes JP, and Dierieck V, et al (2012) Survival benefit of adding hyperthermic intraperitoneal chemotherapy (HIPEC) at the different time-points of treatment of ovarian cancer: review of evidence Curr Pharm Des 18 3793-3803 https://doi. org/10.2174/138161212802002616 PMID: 22591422

21. Bakrin N, Bereder JM, and Decullier E, et al (2013) Peritoneal carcinomatosis treated with cytoreductive surgery and hyperthermic intraperitoneal chemotherapy (HIPEC) for advanced ovarian carcinoma: a French multicentre retrospective cohort study of 566 patients Eur J Surg Oncol 39 1435-1443 https://doi.org/10.1016/j.ejso.2013.09.030 PMID: 24209430

22. Cianci S, Ronsini C, and Vizzielli G, et al (2019) Cytoreductive surgery followed by HIPEC repetition for secondary ovarian cancer recurrence Updates Surg 71 389-394 https://doi.org/10.1007/s13304-018-0600-y

23. Aghajanian C, Blank SV, and Goff BA, et al (2012) OCEANS: a randomized, double-blind, placebo-controlled phase III trial of chemotherapy with or without bevacizumab in patients with platinum-sensitive recurrent epithelial ovarian, primary peritoneal, or fallopian tube cancer J Clin Oncol 30 2039-2045 https://doi.org/10.1200/JC0.2012.42.0505 PMID: 22529265 PMCID: 3646321

24. Oza AM, Cibula D, and Benzaquen AO, et al (2015) Olaparib combined with chemotherapy for recurrent platinum-sensitive ovarian cancer: a randomised phase 2 trial Lancet Oncol 16 87-97 https://doi.org/10.1016/S1470-2045(14)71135-0

25. Mirza MR, Monk BJ, and Herrstedt J, et al (2016) Niraparib maintenance therapy in platinum-sensitive, recurrent ovarian cancer N Engl J Med 375 2154-2164 https://doi.org/10.1056/NEJMoa1611310 PMID: 27717299 
26. Coleman RL, Oza AM, and Lorusso D, et al (2017) Rucaparib maintenance treatment for recurrent ovarian carcinoma after response to platinum therapy (ARIEL3): a randomised, double-blind, placebo-controlled, phase 3 trial Lancet 390 1949-1961 https://doi. org/10.1016/S0140-6736(17)32440-6 PMID: 28916367 PMCID: 5901715

27. Le DT, Uram JN, and Wang H, et al (2015) PD-1 blockade in tumors with mismatch-repair deficiency N Engl J Med 372 2509-2520 https://doi.org/10.1056/NEJMoa1500596 PMID: 26028255 PMCID: 4481136 